\title{
Audit of ankle injuries in an accident and emergency department
}

\author{
G J Packer, C C Goring, A D Gayner, A D Craxford
}

\begin{abstract}
Objective-To determine whether the treatment of ankle injuries in an accident and emergency department could be improved by an audit of existing treatment and the creation and use of a protocol.

Design-The study consisted of three parts: a review of the current treatment and published reports on treatment, the formation of a protocol, and a study of treatment after introducing the protocol.
\end{abstract}

Setting-Accident and emergency department of a district general hospital.

Patients -550 patients attending the department with ankle injuries over four months.

Results - The review of treatment showed that patients with fractures were detected and treated adequately, but most had radiography. Patients with ligamentous injuries may have been undertreated. After introducing the protocol the number of patients undergoing radiography was reduced from $205(80 \%)$ to $186(70 \%)(0.0027<p<0.01)$. In $87 \%$ of the notes reviewed the protocol had been completed. Sixty six patients with ligamentous injuries were reviewed in the department or soft tissue clinic compared with 20 before the protocol was introduced. There was a $53 \%$ reduction in inappropriate referrals to the fracture clinic (13 before $v$ nine after).

Conclusions-Using a protocol can, at little expense, improve the treatment of ankle injuries and reduce the cost of radiology in an accident and emergency department.

Implication-Treatment of other conditions may be improved by introducing a protocol.

\section{Introduction}

Ankle injuries are one of the commonest causes of referral to accident and emergency departments and account for $2 \%$ of all radiographic requests. ${ }^{1}$ Discussion of the treatment of patients with ankle injuries during a regular audit meeting of the orthopaedic and accident and emergency departments had suggested that treatment could be improved to reduce the proportion of patients who had radiography, to reduce the number of patients without fractures who were referred to the fracture clinics, and to ensure adequate treatment and follow up of patients with ligamentous injuries of the ankle. We therefore performed an audit to confirm that these problems existed and to explore ways of improving treatment.

Hospital, Tyne and Wear NE29 8NH G J Packer, FRCSED, registrar in orthopaedic surgery C C Goring, FRCS, consultant in accident and emergency medicine

A D Gayner, FRCS, consultant orthopaedic surgeon

A D Craxford, FRCS,

consultant orthopaedic surgeon

Correspondence to: Mr Packer.

BMF 1991;302:885-7 senior house officers in the department were included in the study. The audit was conducted over 17 weeks corresponding to months 3 to 6 of the house officers' six month attachment to the department. Thus the same officers were present throughout the study. The review of the current treatment and prospective parts of the audit each took eight weeks; the additional week between these two parts was used to produce the algorithm.

The review of the current treatment was performed by identifying, on a weekly basis, patients who had presented with ankle injuries from the accident and emergency register and examining their notes. This review was undertaken without the knowledge of the senior house officers to prevent a change in the way in which patients were treated. At the end of each week the notes were used to assess the signs associated with clinically important ankle injury as well as the number of patients who had radiography and the number who were reviewed. The results of the eight week review were used in association with results in published reports to produce an algorithm. Published reports were searched to identify the best signs of clinically important ankle injury and to allow comparison with current treatment.

The results of our study and the review of published reports, together with the algorithm were presented at a departmental meeting. Discussion resulted in minor changes to the algorithm, which was then introduced for eight weeks. The algorithm was attached to the casualty card by the receptionist when patients presented to the accident and emergency department with ankle injuries.

After the protocol had been in use for eight weeks the casualty notes of patients presenting with ankle injuries were reviewed to assess the protocol's effectiveness. Changes in the proportion of patients undergoing radiography were divided by the standard error of the difference between the proportions to find the probability attached to the change.

\section{Results}

The table shows the results obtained before and after the protocol was introduced. Before the protocol's introduction patients with fractures were identified and treated successfully, but this was achieved by liberal use of radiography ( $80 \%$ of patients). In addition, $46 \%$ of all radiographs were ordered out of hours. The most common criterion for radiography was local ankle tenderness. There were no fractures of the ankle joint missed during this period.

Once fractures had been excluded by radiography, however, only $33(13 \%)$ patients had any form of review. Only one patient was referred to the department's soft tissue clinic despite the fact that this clinic has open access to physiotherapy. Five patients without fractures reattended of their own accord, suggesting that they had been undertreated initially. All the patients who reattended had appreciable swelling of the ankle and had had fractures excluded by radiography. This suggested that they had sustained a ligamentous injury to the ankle.

Published reports suggested that the following features were most closely associated with ankle fractures $^{12}$ : measurable swelling (except when localised to the anterior talofibular ligament), bruising, inability to bear weight, and increasing age. The figure shows the 


\begin{tabular}{|c|c|c|c|c|c|c|c|c|}
\hline & \multirow[b]{2}{*}{$\begin{array}{c}\text { With } \\
\text { ankle } \\
\text { fractures }\end{array}$} & \multirow[b]{2}{*}{$\begin{array}{c}\text { With } \\
\text { other } \\
\text { fracture` }\end{array}$} & \multirow[b]{2}{*}{$\begin{array}{l}\text { Receiving } \\
\text { radiography }\end{array}$} & \multirow[b]{2}{*}{$\begin{array}{l}\text { Receiving } \\
\text { radiography } \\
\text { out of hours }\end{array}$} & \multicolumn{4}{|c|}{ Without fracture and reviewed in: } \\
\hline & & & & & $\begin{array}{l}\text { Accident and } \\
\text { emergency } \\
\text { department }\end{array}$ & $\begin{array}{l}\text { Soft tissue } \\
\text { clinic }\end{array}$ & $\begin{array}{c}\text { Fracture } \\
\text { clinic }\end{array}$ & Total \\
\hline Before protocol $(n=274)$ & 36 & 18 & $205(80)$ & $94(37)$ & 19 & 1 & 13 & $33(13)$ \\
\hline After protocol $(n=276)$ & 32 & 9 & $186(70)$ & $86(32)$ & 55 & 11 & 6 & $72(27)$ \\
\hline
\end{tabular}

^These patients were excluded in calculation of percentages.

algorithm produced for treating ankle injuries. This emphasised the criteria for radiography of ankle injuries and the method of treatment of patients with ligamentous injuries as well as the treatment of ankle fractures.

During the eight week assessment the protocol was completed in $240(87 \%)$ of the notes reviewed. In 17 cases the protocol was not completed and in the remaining 19 no protocol was attached to the notes, either because it had never been attached or because it had become separated by the time of review. The number of patients receiving radiography was reduced by $9 \%(0.0027<\mathrm{p}<0.01)$, and the number of patients reviewed was increased by $18 \%$, most patients being reviewed in the accident and emergency department once to ensure that their symptoms were settling. Referrals to the soft tissue clinic were increased, with a

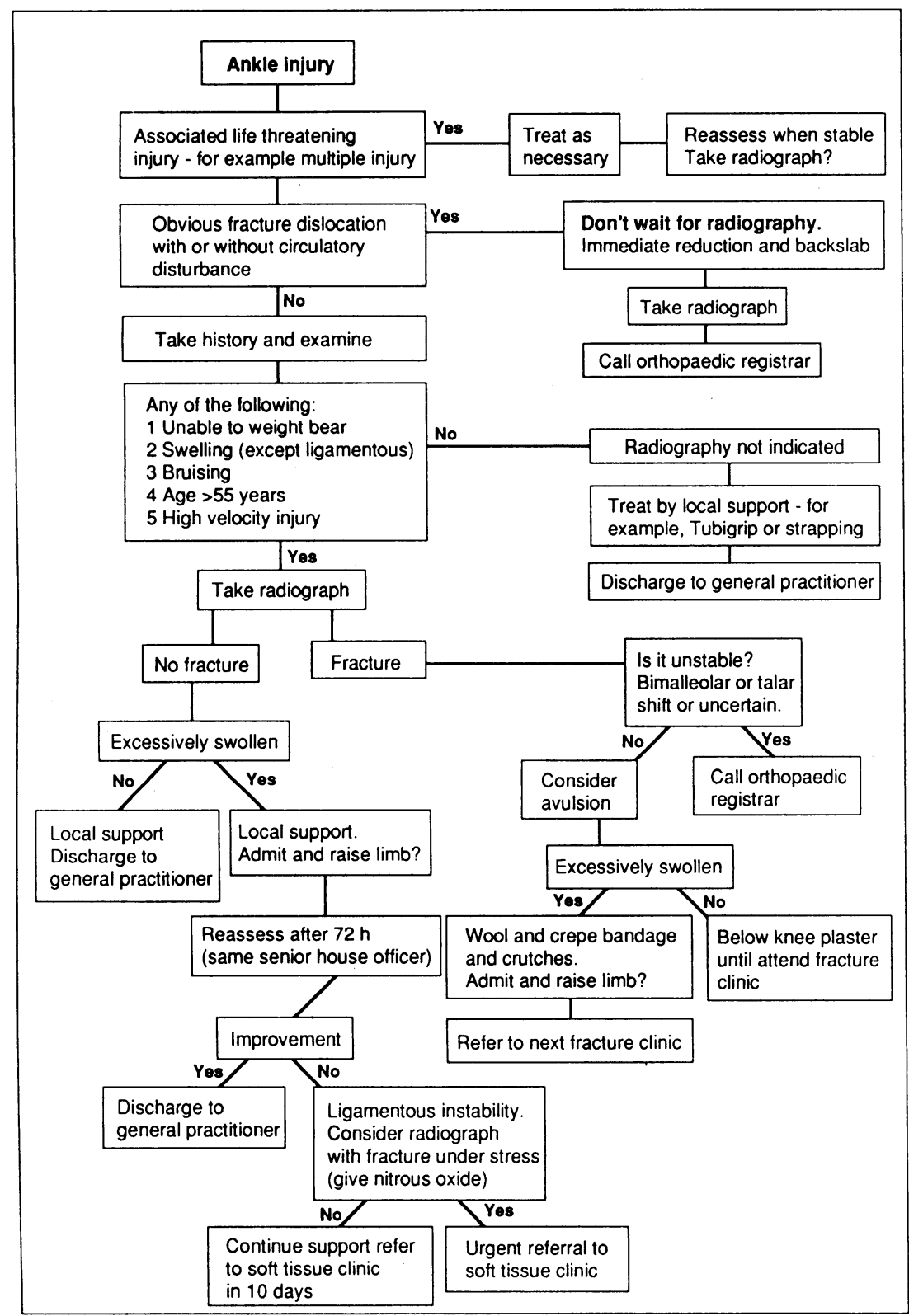

Algorithm for treatment of patients presenting with ankle injuries corresponding reduction in inappropriate referral to fracture clinics. Again there were no missed fractures. Only one patient reattended of his own accord, although two patients who had not undergone radiography (the correct decision according to the protocol) reattended at the request of their general practitioner specifically for radiography (both radiographs showed no fracture).

\section{Discussion}

Introducing monthly departmental audit meetings not only allows formal auditing but offers an opportunity for discussion of conditions that cause confusion or controversy in the department. Having obtained anecdotal evidence of problems we performed a study to confirm that the problems existed and obtain information on treatment to act as a baseline for later comparison.

Months 3-6 of the senior house officers' attachment to the department were chosen for study to ensure that the same doctors took part throughout the study. The first two months were not included to allow time for the officers to gain adequate experience of treating ankle injuries in an accident and emergency department. The review of published reports allowed comparison of the criteria used by house officers in accident and emergency departments to assess the need for radiography. The factor most commonly used was local bony tenderness, which reports suggest is a poor indicator compared with other clinical signs such as swelling and bruising.

Although patients with fractures were identified and adequately treated, our study showed that this was achieved by radiography in most cases. In addition, $46 \%$ of the radiographs were taken out of hours. On call radiography costs almost three times more than that during working hours, principally because of the radiographers' call out fee ( $£ 5.13$ out of hours $v £ 1.79$ day time, excluding any capital costs; radiology department, personal communication).

Most patients who did not have fractures were not reviewed, even though some would undoubtedly have had ligamentous injuries. These patients received no treatment except local support, although controlled studies have shown the benefit of early mobilisation. All the patients who reattended the department of their own accord during the review of current treatment seem to have had ligamentous injuries.

An algorithm was chosen for the protocol because it allowed all the necessary information to be displayed in a form that could easily be attached to each patient's notes. Attaching the algorithm to the case notes not only encouraged the senior house officers to use the protocol but facilitated review. The officers found the algorithm "user friendly" (as shown by the fact that it had been completed in $87 \%$ of the notes reviewed), and it was cheap to produce. The algorithm succeeded in changing the treatment of patients with ankle injuries. Radiography was reduced, with no evidence that fractures were missed as a result, but the percentage of radiographs ordered out of hours remained constant at $46 \%$. Assuming that the number of patients presenting with new ankle injuries each month was about 135 (as it was over the four months studied) and that the 
percentage who underwent radiography out of hours was $46 \%$, then introducing the protocol saved the radiology department almost $£ 500$ a year.

The protocol also resulted in an increase in the number of patients reviewed and hence the work of the accident and emergency department. In terms of the patients reviewed in the department, however, the total numbers (an extra 36 over the eight weeks) were small, amounting to an extra four or five patients a week in a department with an average new patient attendance each week of about 800 .

Eleven of the patients reviewed in the department were referred to the soft tissue clinic, six of whom required physiotherapy. This suggests that, as had been suspected, before the protocol was introduced patients with ligamentous injuries had been undertreated; this seems to justify the small increase in the number of patients reviewed. After introducing the protocol the number of patients reattending of their own accord fell from five to one, supporting the view that the protocol improved the treatment of patients with ligamentous injuries. The protocol also resulted in a reduction of the work of the fracture clinics as inappropriate referrals were reduced by $53 \%$. The two patients who were referred by their general practitioners for radiography show the importance of informing local general practitioners of changes in treatment policy.

At the end of the study no changes were required in the protocol, and the algorithm is now included in the notes of any patient attending with an ankle injury. The algorithm proved an effective means of improving treatment of ankle injuries and algorithms may improve treatment of other conditions in accident and emergency departments.

1 Brooks SC, Potter BT, Rainey JB. Inversion injuries of the ankle: clinical assessment and radiographic review. BMF 1981;282:607-8

2 Montague AP, McQuillan RF. Clinical assessment of apparently sprained ankle and detection of fracture. Injury 1985; 16:545-6.

3 Anonymous. Late consequences of sprained ankle [Editorial]. Lancet 1990;335:

(Accepted l February 1991)

\section{Audit in Person}

\section{Surveys of patient satisfaction: I-Important general considerations}

\section{Ray Fitzpatrick}

\section{Why conduct a survey?}

Discussions about how the quality of health care should be measured increasingly include patient satisfaction as one of the important dimensions. ${ }^{12}$ However, a single explanation of why surveys of patients' views have suddenly become such a visible and regular aspect of the NHS would probably not cite the impact of scientific arguments about the evaluation of health services but the far more influential NHS Management Inquiry. The inquiry crisply and emphatically condemned the failure of the NHS to use the well established techniques of market research to elicit the views and experiences of its users. ${ }^{3}$ The proliferation of surveys that immediately followed that report was largely managerially led and focused on subjects that managers may have felt more competent or confident to tackle, such as the quality of catering and physical amenities provided for inpatients or the accessibility of health care facilities.

Subsequently, important statements from professional bodies argued along similar lines to those of the government's white paper Working for Patients ${ }^{4}$ by underlining the wider contribution of patients' views to assessing quality of care in hospitals and primary care. ${ }^{56}$ It will be unlikely, if these recommendations are heeded, that surveys will continue to concentrate narrowly on so called "hotel" aspects of health care, such as catering. The patient's views will increasingly be sought on such matters as information needs; interpersonal and organisational aspects of care; and, indeed, the value of medical treatments.

There are three reasons besides external pressures from governments, professional bodies, and health authorities why health professionals should take patient satisfaction seriously as a measurement (box). Firstly, there is convincing evidence that satisfaction is an important outcome measure. It may be a predictor of whether patients follow their recommended treatments, ${ }^{\text {? }}$ and is related to whether patients reattend for treatment ${ }^{8}$ and change their provider of health care. ${ }^{9}$ Evidence has also begun to emerge that satisfaction is related to improvements in health status. ${ }^{10}$ "Secondly,

\section{Patient satisfaction as measure of health} care

- An important outcome measure

- Useful in assessing consultations and patterns of communication

- Used systematically, feedback enables choice between alternatives in organising or providing health care

patient satisfaction is an increasingly useful measure in assessing consultations and patterns of communication (such as the success of giving information, of involving the patient in decisions about care, and of reassurance). ${ }^{12}$ Thirdly, patient feedback can be used systematically to choose between alternative methods of organising or providing health care (such as length of consultation or arrangements for out of hours care). ${ }^{13}$

Health professionals remain largely unfamiliar with methods of measurement derived from survey research. This paper considers some of the potential problems and strategic questions involved in surveys of patient satisfaction. A subsequent article will examine some of the main considerations involved in designing, conducting, and analysing a survey of patients' views.

\section{Why not to conduct a survey}

The time, resources, and staff required to design, conduct, and analyse a survey are invariably underestimated. Given that various costs are associated with even the most modest survey, alternative methods of obtaining the desired information or goal should always be seriously considered. For example, in general terms we already know much about many of the matters most commonly associated with patient dissatisfaction. ${ }^{14}$ With regard to hospital care, well established complaints would include waiting for an outpatient appointment or admission, waiting at clinics, and inadequate or poor information at all stages 